\title{
Mutual Coupling Reduction for Dual-Band MIMO Antenna with Simple Structure
}

\author{
Chao-ming LUO, Jing-song HONG, Muhammad AMIN \\ School of Physical Electronics, University of Electronic Science and Technology of China, No. 4, Section 2 , \\ Chengdu 610054, China
}

461339852@163.com, cemlab@uestc.edu.cn, aminssphysics@hotmail.com

Submitted October 22, 2016 / Accepted February 16, 2017

\begin{abstract}
In this paper, a novel dual-band MIMO (multiple input, multiple output) antenna for WLAN (wireless local area network) applications is presented. The MIMO antenna contains two dual-band antenna elements, each of which comprises a T-shaped monopole and a special Fshaped stub resonator. Two operating bands with center frequencies of $5.5 \mathrm{GHz}$ and $2.5 \mathrm{GHz}$ are crested by the monopole of $T$ shape and the stub resonator of 1 shape, accordingly. The --shaped stub also works as an isolation structure at the higher band, which can simplify the dualband isolation design into a single-band problem. Moreover, the isolation is enhanced at the lower band by inserting a metal strip which can cancel out original coupling. The inserted metal strip is the only additional decoupling structure in this design and has a simple texture with a compact size. The measured and simulated results reveal that the designed MIMO antenna can cover all the 2.4/5.2/5.8 GHz WLAN operating bands and within the recommended bands the isolations exceed by $20 \mathrm{~dB}$.
\end{abstract}

\section{Keywords}

Dual-band, high isolation, multiple-input multipleoutput (MIMO) antenna, wireless local area network (WLAN)

\section{Introduction}

Multiple-input and multiple output (MIMO) technology has attracted more research attention due to the demands for higher transmission rate and more reliable link in wireless communications. MIMO is a complex technology by utilizing multiple antennas to increase the channel capacity and overcome multipath fading propagation problem [1], [2]. However, due to the space limit at the sizeshrinking terminal devices, the most critical problem in a MIMO antenna design is how mutual coupling can be reduced between the closely packed antenna elements.

Various studies had been performed to boost the isolation of the antenna ports [3-13]. A tree-like parasitic structure [3], or mushroom like EBG structure [4-6] can minimize the mutual coupling among radiating components though restraining the surface wave propagation. The defected ground structure [7] or a simple ground plane modification [8] performing as a band-stop filter was designed to increase the isolation. Use of slot has been useful to include a notch in the isolation between antennas [9-11]. In [12], a method for isolation enhancement, based on metamaterial was given for the MIMO antenna. However, these methods have the common deficiency of complex structures, which will occupy a large space on the antenna. In [13], a neutralization line is physically linked to the antenna components for improving isolation. In this structure, the exiting mutual currents on the sufferer antenna are counterbalance by implanting $180^{\circ}$ out-of-phase currents from an excitation antenna. The neutralization-line technique is extensively used as it occupies small space. However, this method markedly deteriorates the reflection coefficient and therefore, the trial and error process are required to obtain the suitable dimensions which need lots of time.

Moreover, most decoupling methods as mentioned above are only suited for offering narrow band isolation among two antennas. In previous studies on dual-band MIMO antennas [14-17], mutual coupling reduction is usually achieved by two isolation structures. One is used for lower band, while the other one is for higher band. However, combination of the two isolation structures may increase complexity of system, especially when the isolation structures are complex.

In this design, a novel structure of the antenna element is applied, which can avoid this problem. The dualband antenna element comprises a monopole of $\mathrm{T}$ shape and a stub resonator of special $\vdash$ shape. At the lower frequencies, the F-shaped stub can be considered as an antenna because it is coupled-fed by the T-shaped monopole. At the higher frequencies, it can reduce mutual coupling though suppressing surface wave propagation, which works as a reflective component. Thus, the space of the antenna is maximum utilized and the design of dual-band MIMO antenna is simplified to a single-band problem, which only needs to consider enhancing isolation at the 
lower band. Moreover, instead of adding a neutralization line that is physically joined to the antenna elements [13], we put a metal strip between the antenna elements in order to artificially generate a supplementary coupling route for enhancing the isolation. The metal strip, which is the only additional decoupling structure, has small effect on initial antenna impedance as it is not physically connected to the antenna. Due to the dual function of 1 -shaped stub and the small occupied space of the metal strip, this antenna has a small size and simple structure.

\section{Antenna Structure}

The geometry of the proposed dual-band MIMO antenna can be seen in Fig. 1. The antenna is printed on a $26 \times 50 \mathrm{~mm}^{2}$ FR4 substrate. This substrate has thickness of $0.8 \mathrm{~mm}$ with relative dielectric constant of 4.4 and loss tangent of 0.02 . The MIMO antenna contains of two T-shaped monopoles, two $\vdash$-shaped grounded branches and a metal strip. A T-shaped monopole and a $\vdash$-shaped stub compose a dual-band antenna component and the two antenna components are printed symmetrically. The desirable high isolation is obtained by the metal strip and the unique structure of the antenna element. In the following, more details are studied.

\section{Antenna Design}

\subsection{Dual-band Antenna Element}

In Fig. 2(a), the composition of an element of the dual-band antenna can be seen. The antenna is made of a monopole of $\mathrm{T}$ shape and a special stub resonator of $\vdash$ shape, which are engraved on the substrate at the different surfaces. A $50-\Omega$ microstrip line is used to directly excite the T-shaped monopole, whereas the stub resonator having $\vdash$ shape extended from ground is coupled-fed from the driven the T-shaped monopole. In Fig. 2(b), the simulated $\mathrm{S}_{11}$ of the antenna component without and with the

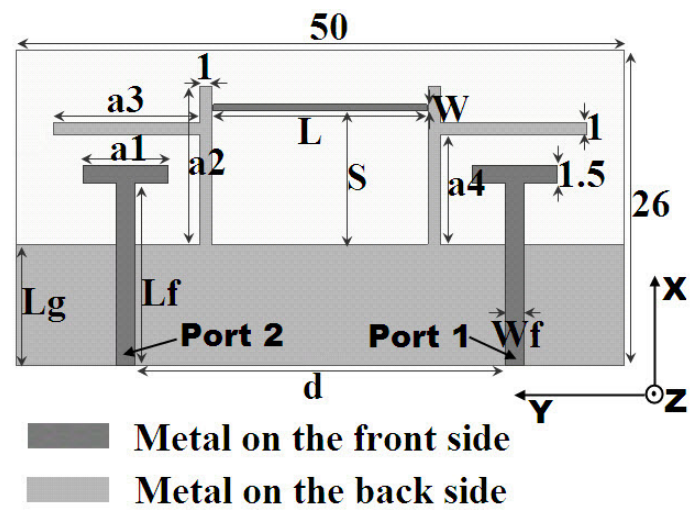

Fig. 1. Geometry of the dual-band MIMO antenna: $L_{\mathrm{g}}=10$, $L_{\mathrm{f}}=15, \quad W_{\mathrm{f}}=1.5, \quad a_{1}=7, \quad a_{2}=13, \quad a_{3}=12, \quad a_{4}=9$, $d=30.5, \quad L=17.6, \quad S=11, \quad$ and $\quad W=0.5$. (All dimensions are in millimeters). resonator stub having $\vdash$ shape is shown. As observed, the T-shaped monopole without $\vdash$-shape stub has an operating frequency at around $5.5 \mathrm{GHz}$. The $\vdash$-shaped stub provides an additional resonance frequency of $2.5 \mathrm{GHz}$ and improves the impedance matching characteristic from 5 to $6 \mathrm{GHz}$. Moreover, the current distributions for the two operating frequencies can be seen in Fig. 3. As inspected, the main currents are on the T-shaped monopole at $5.5 \mathrm{GHz}$, while the stronger currents are distributed near the $\vdash$-shaped stub at $2.5 \mathrm{GHz}$. Thus, we can conclude that, the T-shaped monopole determines the resonance of the upper frequency band at $5.5 \mathrm{GHz}$, while at the lower frequency $2.5 \mathrm{GHz}$, the $\vdash$-shaped stub regulates the impedance matching.

For better illustrating, the parametric studies are plotted in Fig. 4. As observed in Fig. 4, increasing lengths of the resonant structures cause the operating band to shift to lower frequencies. The optimal length of the resonant structures is approximately equivalent to a quarter-wavelength at the corresponding frequency. The concept of using a parasitic element has been also useful to design multiband monopole antennas [18]. Figure 5 shows the simulated radiation patterns of the antenna element. As suspected, the cross polarization is relatively high because of the introducing of the horizontal segment of the $\vdash$-shaped stub. This is not a serious problem for WLAN applications since the propagation channels are usually multipath, especially in the indoor environment.

The F-shaped stub extended from ground works as a main radiator of the antenna at the $2.4-2.6 \mathrm{GHz}$ band, while it works as a reflective component at other bands. This indicates that when the proposed dual-band antenna

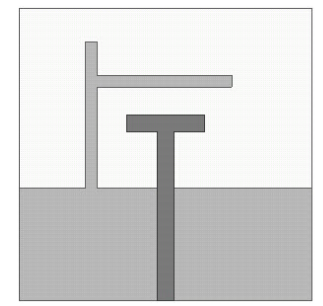

(a)

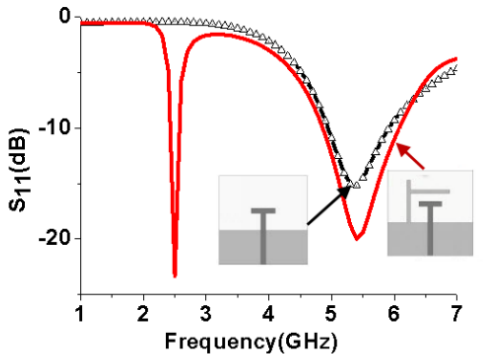

(b)
Fig. 2. (a) Geometry and (b) $\mathrm{S}_{11}$ of dual-band antenna element.

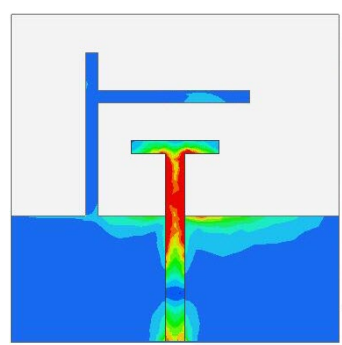

(a)

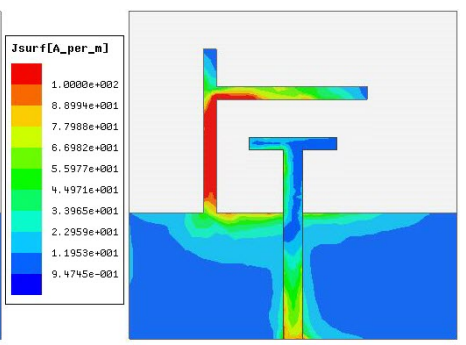

(b)
Fig. 3. Simulated, surface current distributions at (a) $2.5 \mathrm{GHz}$ and (b) $5.5 \mathrm{GHz}$ 


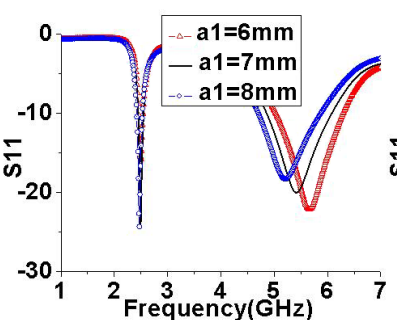

(a)

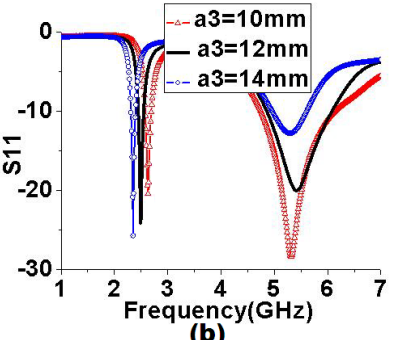

(b)
Fig. 4. Simulated $\mathrm{S}_{11}$ for different values of (a) $a 1$ and (b) $a 3$.

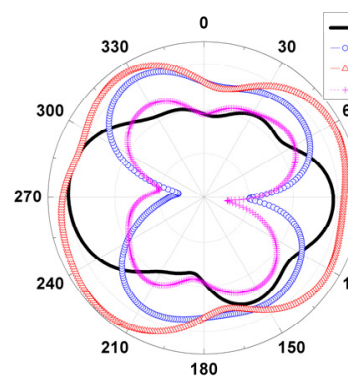

(a)

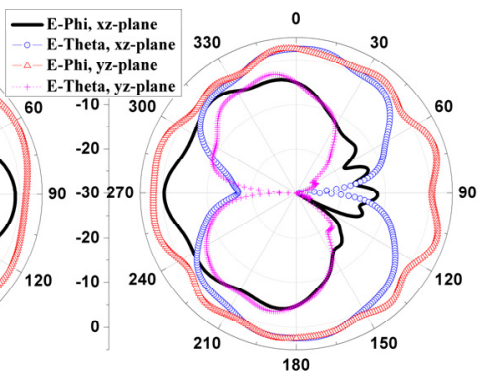

(b)
Fig. 5. Simulated radiation patterns of dual-band antenna element at (a) $2.5 \mathrm{GHz}$ and (b) $5.5 \mathrm{GHz}$.

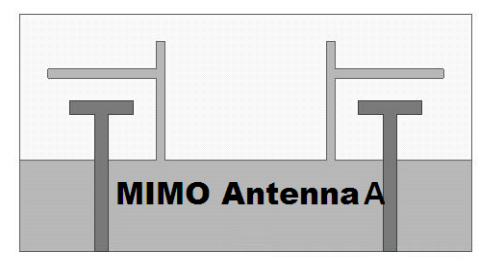

(a)

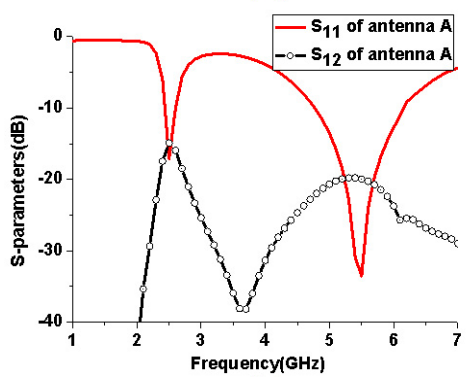

(b)

Fig. 6. (a) Geometry, (b) $S_{11}$ and $S_{12}$ of MIMO antenna A.

component is operating to construct a two-component MIMO antenna structure (MIMO Antenna A), a good isolation may be obtained at the higher frequencies 5-6 GHz. It can be proved by the S-parameters of antenna A, as shown in Fig. 6. The $\mathrm{S}_{12}$ is lower than $-20 \mathrm{~dB}$ in the frequency range $5-6 \mathrm{GHz}$ and $-15 \mathrm{~dB}$ over $2.4-2.6 \mathrm{GHz}$. Moreover, in both the upper band $(5-6 \mathrm{GHz})$ and lower band $(2.4-2.6 \mathrm{GHz})$, the $\mathrm{S}_{11}$ is lower than $-10 \mathrm{~dB}$.

\subsection{Metal Strip}

As mentioned above, high isolation can be accomplished without any additional decoupling structures for the upper band and that is because of the unique structure of the antenna component. In order to lessen the mutual coupling for the lower band, a metal strip of size $17.6 \times 0.5 \mathrm{~mm}^{2}$ is placed between the elements, which can introduce new coupling to counteract the initial coupling. Different from the traditional neutralization line, which markedly deteriorates the reflection coefficient, the metal strip in this design is not physically connected to the antenna elements and is perpendicular to the microstrip line. To understand the working mechanism deeply, parameter studies are carried out. The simulated S-parameters for different $L$ and $S$ are shown in Figs. 7 and 8, respectively. For clarity, these figures for $\mathrm{S}_{12}$ have been enlarged around the frequency of $2.5 \mathrm{GHz}$. Figures 7(a) and 8(a) show that $\mathrm{S}_{11}$ varies less with the parameters, indicating a minor

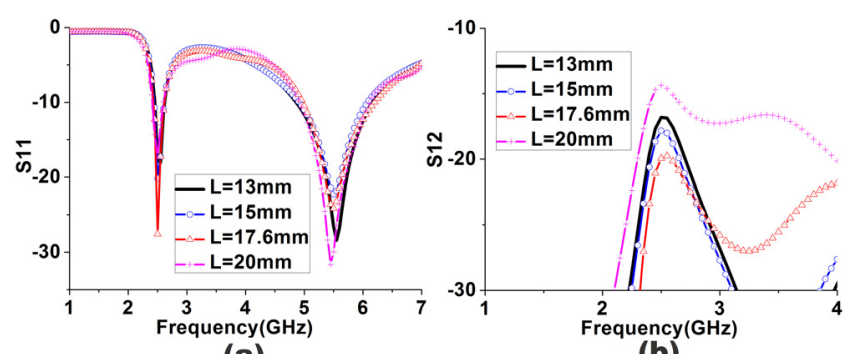

(a)

(b)

Fig. 7. S-parameters with different lengths of the metal strip: (a) $\mathrm{S}_{11}$ (b) $\mathrm{S}_{12}$.

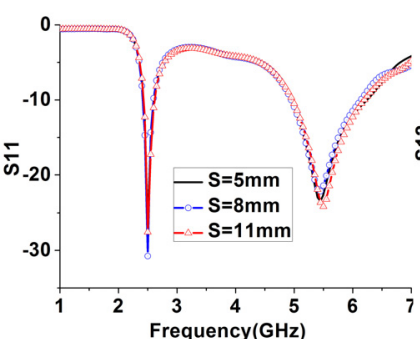

(a)

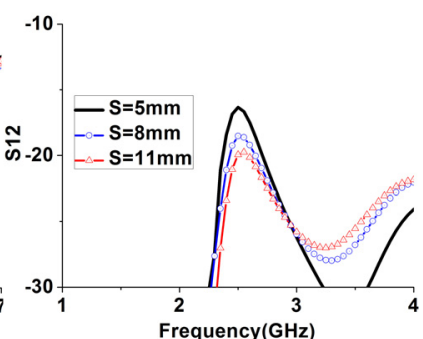

(b)
Fig. 8. S-parameters with different positions of the metal strip: (a) $S_{11}$ (b) $S_{12}$.

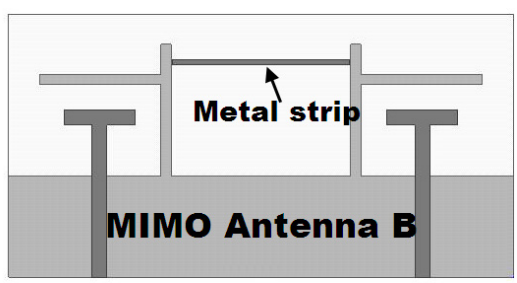

(a)

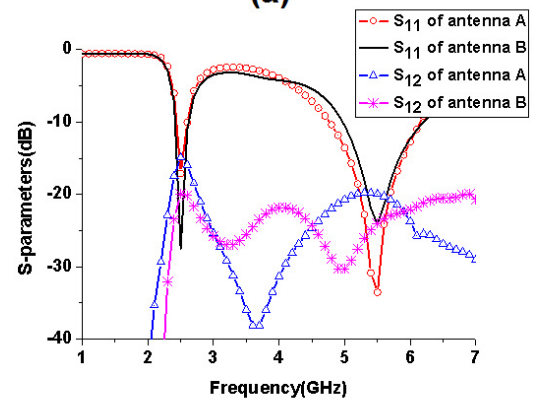

(b)

Fig. 9. (a) Structure of MIMO antenna $B$, (b) $S_{11}$ and $S_{12}$ of antenna A and B. 


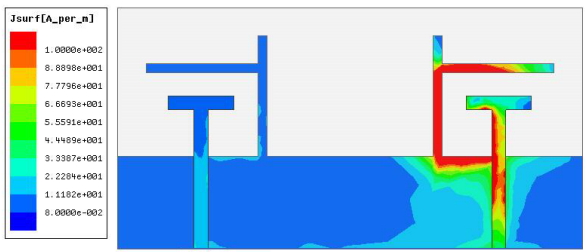

(a)

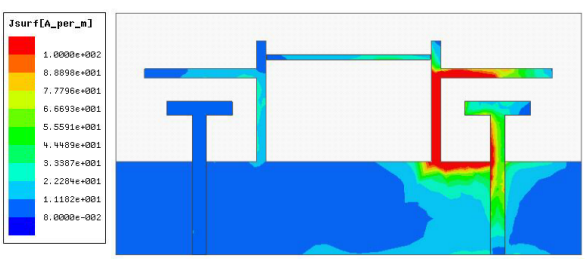

(b)

Fig. 10. At $2.5 \mathrm{GHz}$, current distributions on MIMO (a) A and (b) B antennas.

effect of the metal strip on reflection coefficient. As shown in Figs. 7(b) and 8(b), when $L$ increases from $13 \mathrm{~mm}$ to $17.6 \mathrm{~mm}$ or $S$ varies from $5 \mathrm{~mm}$ to $11 \mathrm{~mm}$, the isolation at $2.5 \mathrm{GHz}$ is improved about $5 \mathrm{~dB}$. The reason for this phenomenon is that the additional coupling produced by the metal strip is insufficient if the metal strip is short or $S$ is small. As expected, when the metal strip is too long ( $L=20 \mathrm{~mm}$ ), the isolation gets worse caused by excessive additional coupling. Moreover, at other frequencies, the metal strip also introduces more or less coupling to change the isolation characteristics, but remains within an acceptable range i.e. higher than $20 \mathrm{~dB}$.

In Fig. 9, the return losses of antenna A (without the metal strip) and antenna B (with the metal strip) are given. The mutual coupling is reduced to less than $-20 \mathrm{~dB}$ over the entire WLAN band by adding the metal strip within the two antenna elements. It can also be observed that the effect of the metal strip on $\mathrm{S}_{11}$ is small. The mechanism of decoupling is that the metal strip yields a supplementary route for the coupling currents, and the original coupling should be mostly offset. The currents from the excited antenna component to the non-excited component are significantly reduced, it is shown in Fig. 10.

\section{Experimental Results}

In order to verify the results obtained from simulations, a prototype antenna was fabricated and tested, which can be seen in Fig. 11.

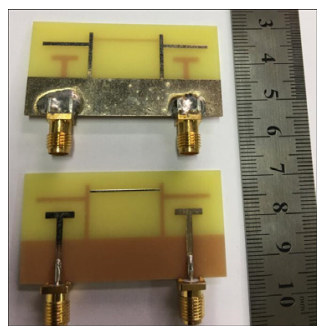

Fig. 11. Photograph of the fabricated antenna.

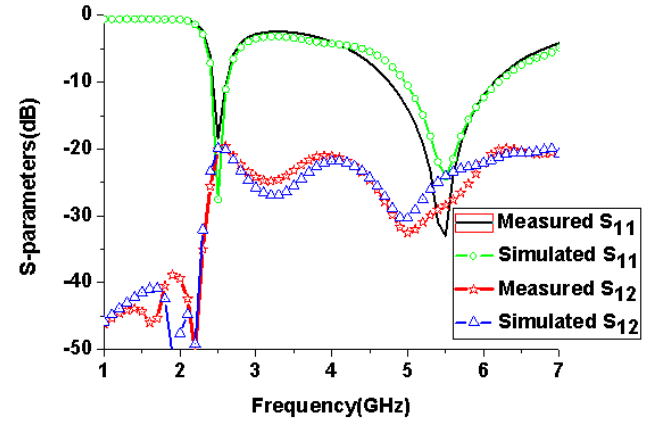

Fig. 12. $S_{11}$ and $S_{12}$ of the antenna (proposed).

Figure 12 shows the measured and simulated Sparameters. The measured $-10 \mathrm{~dB}$ impedance bandwidth for the $2.4 \mathrm{GHz}$ is $300 \mathrm{MHz}$ (from $2.35 \mathrm{GHz}$ to $2.65 \mathrm{GHz}$ ) band and for the $5.2 / 5.8 \mathrm{GHz}$ bands is $1.3 \mathrm{GHz}$ (from 4.9 to $6.2 \mathrm{GHz}$ ). For the higher and lower frequency bands, the measured mutual couplings are lower than $-26 \mathrm{~dB}$ and $-20 \mathrm{~dB}$, respectively. It can be confirmed by the nearness of the measured and simulated frequency responses of the fabricated antenna that the operating band satisfies the design demands.

For evaluating the proposed MIMO antenna functioning, the (ECC) envelope correlation coefficient, a measure to describe how much the communication channels are isolated with each other, is taken as an analytical parameter. It can be calculated from the S-parameters using the following formula [19]:

$$
E C C=\frac{\left|S_{11}^{*} S_{12}+S_{21}^{*} S_{22}\right|^{2}}{\left|\left(1-\left|S_{11}\right|^{2}-\left|S_{21}\right|^{2}\right)\left(1-\left|S_{22}\right|^{2}-\left|S_{12}\right|^{2}\right)\right|} .
$$

The measured and simulated ECCs across the desired frequency bands are shown in Fig. 13. It can be seen that both the ECCs of the proposed MIMO antenna are below 0.06 over the complete WLAN frequency band, which is much lower than criterion of low ECC $(\mathrm{ECC}<0.5)[19]$. In addition, equation (1) illustrates that small $\left|\mathrm{S}_{11}\right|$ and $\left|\mathrm{S}_{12}\right|$ can cause low ECC. It can be inferred that desired ECC demands good performances in impedance matching and isolation. With the low return loss and high isolation, the ECC of the proposed MIMO antenna is almost 0 at the operating bands $(2.35 \mathrm{GHz}$ to $2.65 \mathrm{GHz}$, and $4.9 \mathrm{GHz}$ to $6.2 \mathrm{GHz}$ ). The difference in simulated and measured ECCs

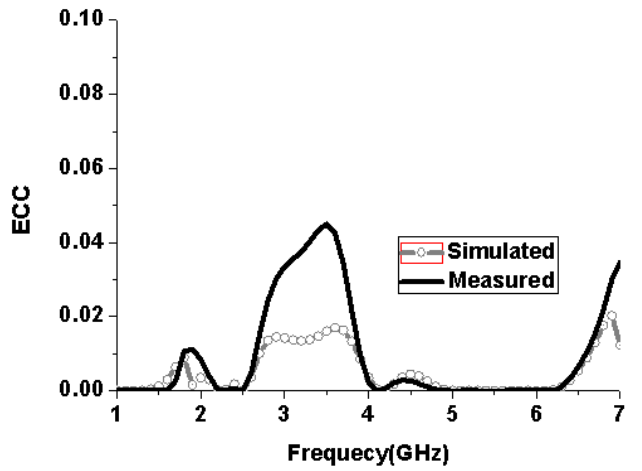

Fig. 13. Simulated and measured ECC. 


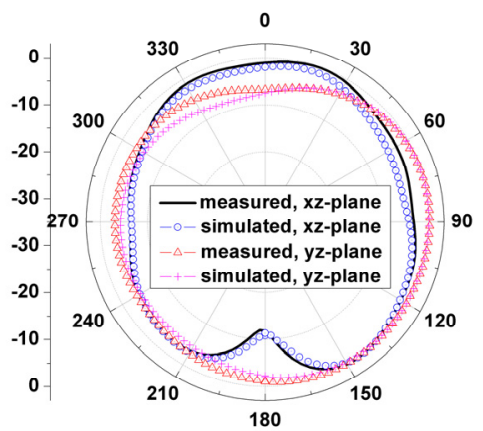

(a)

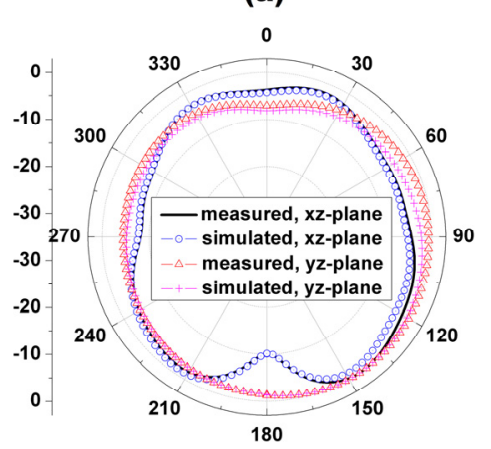

(b)

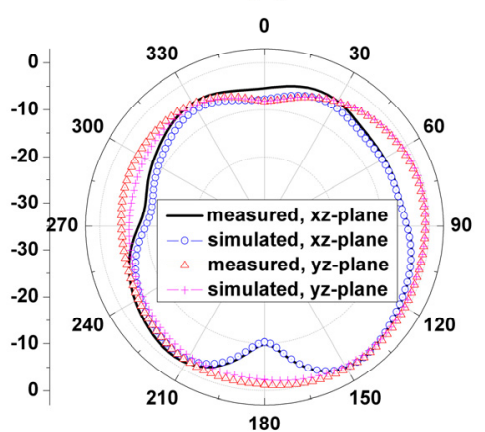

(c)

Fig. 14. Radiation patterns of the proposed MIMO antenna at (a) $2.4 \mathrm{GHz}$, (b) $5.2 \mathrm{GHz}$, and (c) $5.8 \mathrm{GHz}$.

in the notched bands is relatively large. That is because in the case of mismatch, the isolation plays a major role and has a large proportion in ECC.

In Fig. 14, the measured normalized radiation patterns of the three frequencies $2.4,5.2$, and $5.8 \mathrm{GHz}$ for the proposed (MIMO) antenna are plotted. For measurements, during the excitation of port1, port 2 is cut off with a matched load of $50 \Omega$. The radiation patterns were measured for the total electric-field. It can be seen that, in $y-z$ plane the antenna is omnidirectional and near-omnidirectional in $x-z$ plane, which can receive the signals from all the directions. In addition, at the frequencies of 2.4, 5.2, and $5.8 \mathrm{GHz}$, the peak gains reach $1.56,4.43$, and $4.7 \mathrm{dBi}$, respectively, with the corresponding efficiencies of $70.1 \%$, $82.3 \%$, and $81.2 \%$.

\section{Conclusions}

The basic idea of this design is the development of a decoupling method for dual-band with a simple and com- pact geometry. In this article, a dual-band (MIMO) antenna for WLAN applications is exposed. A novel monopole element is used to provide high isolation for higher band, where radiating element and the isolation structure share the same structure. Moreover, the metal strip, which is the only additional decoupling structure, has occupied a small space and produces a significant enhancement of isolation at lower band.

\section{Acknowledgments}

This work was supported by the National Natural Science Foundation of China (No. 61172115 and No. 60872029), the High-Tech Research and Development Program of China (No. 2008AA01Z206), the Aeronautics Foundation of China (No. 20100180003), the Fundamental Research Funds for the Central Universities (No. ZYGX2009J037), Project 9140A07030513DZ02098 and the Fundamental Research Funds for the Central Universities (Grant No. ZYGX2012YB019).

\section{References}

[1] FOSCHINI, G. J., GANS, M. J. On limits of wireless communications in a fading environment when using multiple antennas. Wireless Personal Communications, 1988, vol. 6, no. 3, p. 311-335. DOI: $10.1023 / \mathrm{A}: 1008889222784$

[2] ZHENG, L., TSE, D.N.C. Diversity and multiplexing: A fundamental tradeoff in multiple-antenna channels. IEEE Transactions on Information Theory, 2003, vol. 49, no. 5, p. 1073 to 1096 . DOI: $10.1109 /$ TIT.2003.810646

[3] ZHANG, S., YING, Z., XIONG, J., et al. Ultrawideband $\mathrm{MIMO} /$ diversity antennas with a tree-like structure to enhance wideband isolation. IEEE Antennas Wireless and Propagation Letters, 2009, vol. 8, p. 1279-1282. DOI: 10.1109/LAWP.2009.2037027

[4] COUlombe, M., KOOdiAni, S. F., CAloz, C. Compact elongated mushroom (EM)-EBG structure for enhancement of patch antenna array performances. IEEE Transactions on Antennas and Propagation, 2010, vol. 58, no. 4, p. 1076-1086. DOI: 10.1109/TAP.2010.2041152

[5] YANG, F., RAHMAT-SAMII, Y. Microstrip antennas integrated with electromagnetic band-gap EBG structures: A low mutual coupling design for array applications. IEEE Transactions on Antennas and Propagation, 2003, vol. 51, no. 10, p. 2936-2946. DOI: 10.1109/TAP.2003.817983

[6] GHOSH, S., TRAN, T., LE-NGOC, T. Dual-layer EBG-based miniaturized multi-element antenna for MIMO systems. IEEE Transactions on Antennas and Propagation, 2014, vol. 62, no. 8, p. 3985-3997. DOI: 10.1109/TAP.2014.2323410

[7] SHARAWI, M. S., NUMAN, A. B., KHAN, M. U., et al. A dualelement dual-band MIMO antenna system with enhanced isolation for mobile terminals. IEEE Antennas and Wireless Propagation Letters, 2012, vol. 11, p. 1006-1009. DOI: 10.1109/LAWP.2012.2214433

[8] LI, H., XIONG, J., HE, S. A compact planar MIMO antenna system of four elements with similar radiation characteristics and isolation structure. IEEE Antennas and Wireless Propagation 
Letters, $\quad 2009, \quad$ vol. $\quad 8, \quad$ p. $\quad 1107-1110 . \quad$ DOI: 10.1109/LAWP.2009.2034110

[9] PARK, J., CHOI, J., PARK, J. Y., et al. Study of a T-shaped slot with a capacitor for high isolation between MIMO antennas. IEEE Antennas and Wireless Propagation Letters, 2012, vol. 11, p. 1541-1544. DOI: 10.1109/LAWP.2012.2226695

[10] ANDUJAR, A., AUGUERA, J. MIMO multiband antenna system combining resonant and nonresonant elements. Microwave and Optical Technology Letters, 2014, vol. 56, no. 5, p. 1076-1084. DOI: $10.1002 / \mathrm{mop} .28282$

[11] ANDUJAR, A., AUGUERA, J. MIMO multiband antenna system with nonresonant elements. Microwave and Optical Technology Letters, 2015. vol. 57, no. 1, p. 183-190. DOI: 10.1002/mop.28810

[12] HSU, C., LIN, K., SU, H. Implementation of broadband isolator using metamaterial-inspired resonators and a $\mathrm{T}$-shaped branch for MIMO antennas. IEEE Transactions on Antennas and Propagation, 2011, vol. 59, no. 10, p. 3936-3939. DOI: 10.1109/TAP.2011.2163741

[13] SU, S., LEE, C., CHANG, F. Printed MIMO-antenna system using neutralization-line technique for wireless USB-dongle applications. IEEE Transactions on Antennas and Propagation, 2012, vol. 60 , no. 2, p. 456-463. DOI: 10.1109/TAP.2011.2173450

[14] SU, H., HSU, C., LIN, K. Dual-band insulator design by stacking capacitively loaded loops for MIMO antennas. Electronics Letters, 2010, vol. 46, no. 20, p. 1364-1365. DOI: 10.1049/el.2010.1756

[15] LI, G., ZHAI, H., MA, Z., et al. Isolation-improved dual-band MIMO antenna array for LTE/WiMAX mobile terminals. IEEE Antennas and Wireless Propagation Letters, 2014, vol. 13, p. 1128-1131. DOI: 10.1109/LAWP.2014.2330065

[16] LI, L., HUO, F., JIA, Z., et al. Dual zeroth-order resonance antennas with low mutual coupling for MIMO communications. IEEE Antennas and Wireless Propagation Letters, 2013, vol. 12, p. 1692-1695. DOI: 10.1109/LAWP.2013.2294686

[17] ZHAO, W., LIU, L., CHEUNG, S. W., et al. Dual-band MIMO antenna using double-T structure for WLAN applications. In 2014 International Workshop on Antenna Technology: Small Antennas, Novel EM Structures and Materials, and Applications (iWAT). Sydney (Australia), 2014, p. 232-235. DOI: 10.1109/IWAT.2014.6958646

[18] RISCO, S., ANGUERA, J., ANDUJAR, A., et al. Coupled monopole antenna design for multiband handset devices.
Microwave and Optical Technology Letters, 2010, vol. 52, no. 2, p. 359-364. DOI: $10.1002 /$ mop. 24893

[19] YAN, S., SOH, P. J., VANDENBOSCH, G. A. E. Dual-band textile MIMO antenna based on substrate-integrated waveguide (SIW) technology. IEEE Transactions on Antennas and Propagation, 2015, vol. 63, no. 11, p. 4640-4647. DOI: 10.1109/TAP.2015.2477094

\section{About the Authors ...}

Chao-ming LUO was born in Jingdezhen, China. He received his B.S. degree from ChengDu University of Information Technology in 2009. He is now working towards his Ph.D. degree in Radio Physics at the University of Electronic Science and Technology of China (UESTC). His interests include antenna technology and wireless communication technology.

Jing-song HONG received the B.S. degree in Electromagnetic from LanZhou University, China, in 1991, and the M.S. and Ph.D. degrees in Electrical Engineering from the University of Electronic Science and Technology of China (UESTC), in 2000 and 2005, respectively. He is now a professor with UESTC. His research interests include the use of numerical techniques in electromagnetic and the use of microwave methods for materials characterization and processing.

Muhammad AMIN was born in D.I.Khan, Pakistan. He received his Master degree from Gomal University D.I.Khan Pakistan in 2003.After that he worked at weather surveillance Radar D.I.Khan till 4th March 2011. Then he taught Physics in the Education Department KPK Pakistan till August 2014. Since September 2014 he is doing his Ph.D degree in Radio Physics at the University of Electronic Science and Technology of China (UESTC). His interests include antenna technology and wireless communication technology. 